\title{
Monitoring cell-cell contacts in vivo in transgenic animals
}

\author{
Ting-Hao Huang ${ }^{1,2}$, Tarciso Velho ${ }^{1,3}$ and Carlos Lois ${ }^{1, *}$
}

\begin{abstract}
We used a synthetic genetic system based on ligand-induced intramembrane proteolysis to monitor cell-cell contacts in animals. Upon ligand-receptor interaction in sites of cell-cell contact, the transmembrane domain of an engineered receptor is cleaved by intramembrane proteolysis and releases a protein fragment that regulates transcription in the interacting partners. We demonstrate that the system can be used to regulate gene expression between interacting cells, both in vitro and in vivo, in transgenic Drosophila. We show that the system allows for detection of interactions between neurons and glia in the Drosophila nervous system. In addition, we observed that when the ligand is expressed in subsets of neurons with a restricted localization in the brain it leads to activation of transcription in a selected set of glial cells that interact with those neurons. This system will be useful to monitor cell-cell interactions in animals, and can be used to genetically manipulate cells that interact with one another.
\end{abstract}

KEY WORDS: Interacting cells, Development, Adhesion, Morphogenesis, Cell communication

\section{INTRODUCTION}

Cell-cell contacts are fundamental to the development and function of multicellular organisms. Cell-cell interactions are crucial, for example, for the specification of embryonic tissues, the maintenance of stem cell niches, and cell migration. Neuronal cells form a specialized type of cell-cell interaction, the synapse, which enables the transmission of information through brain circuits. In addition, abnormal cell-cell interactions are fundamental to the pathogenesis of several diseases, most notably to the escape of metastatic cells from tumors (Wang et al., 2005).

There are few methods currently available to analyze cell-cell contacts, most of them designed to study interactions between neurons. The most commonly used methods are colocalization of fluorescent tags by light microscopy, serial electron microscopy (Denk and Horstmann, 2004), GRASP (GFP reconstitution across synapses) (Feinberg et al., 2008) and intercellular transport of viruses (Enquist and Card, 2003; Wickersham et al., 2007). Each of these methods has its strengths and weaknesses, but, importantly, none of these methods enables long-term genetic modifications of the interacting cells.

Recently, a number of genetically encoded strategies have been developed to monitor cell-cell interactions based on the Notch-Delta

${ }^{1}$ California Institute of Technology, Division of Biology and Biological Engineering Beckman Institute MC 139-74, 1200 East California BIvd, Pasadena, CA 91125 , USA. ${ }^{2}$ Department of Neurobiology, University of Massachusetts Medical School, Worcester, MA 01655, USA. ${ }^{3}$ Brain Institute, Federal University of Rio Grande do Norte, Natal, RN 59056-450, Brazil.

*Author for correspondence (clois@caltech.edu)

iD C.L., 0000-0002-7305-2317

Received 19 July 2016; Accepted 13 September 2016 mechanism (Gordon et al., 2015; Morsut et al., 2016; Roybal et al., 2016) or variations thereof (Barnea et al., 2008; Jagadish et al., 2014). The Notch/Delta pathway controls cell fate during development through cell-cell interactions (Artavanis-Tsakonas and Muskavitch, 2010). Delta is one of the ligands for Notch receptors (other ligands in vertebrates are Jagged and Serrate) (Kopan and Ilagan, 2009). Previous works have demonstrated that the key element controlling the activation of the Notch receptor is the Notch regulatory region (NRR), a 200 aa fragment of the Notch extracellular domain (ECD) located immediately before the transmembrane domain (TMD) (Gordon et al., 2007). The NRR is folded in such a way that, in the absence of Delta binding, a cleavage site (called S2) is inaccessible to the action of ubiquitous metalloproteases such as Kuzbanian (in Drosophila) or TACE (tumor necrosis factor- $\alpha$-converting enzyme, or ADAM17, in vertebrates) (Tiyanont et al., 2011). Upon Delta-Notch binding, it is hypothesized that the NRR partially unfolds, thereby increasing the accessibility of the S2 site, and the NRR can then be cleaved by the metalloproteases (Stephenson and Avis, 2012; Tiyanont et al., 2011; Meloty-Kapella et al., 2012; Gordon et al.; 2015). After S2 cleavage, a subsequent cleavage (called S3) by the ubiquitous metazoan gamma-secretase complex occurs in the TMD, within the cell membrane (Brou et al., 2000; Mumm et al., 2000). After S3 cleavage, the intracellular domain (ICD) of Notch loses its membrane anchorage and translocates to the nucleus where it regulates transcription of cell fate-related genes (Struhl and Adachi, 1998, 2000) (Fig. 1A).

Previous experiments have demonstrated that the Notch receptor can be engineered by maintaining the ECD and TMD of the native Notch but replacing the ICD with artificial gene regulatory modules such as the transcriptional activator Gal4 (Struhl and Adachi, 2000; Sprinzak et al., 2010) or the recombinase Cre (Vooijs et al., 2007). These experiments demonstrate that the molecular mechanism of the Delta-Notch system can be used to monitor cell-cell interactions. However, the Notch-Delta system cannot be used in this way in animals without further modifications because it is highly conserved throughout the animal kingdom, and it is likely that there could be cross-talk between Notch and Delta ECDs from different species. Thus, exogenous expression of the native ECDs of Notch or Delta is problematic because it may severely perturb development due to dominant-negative effects of the engineered ligands and receptors. In addition, the potential interaction between endogenous Delta (plus Jagged and Serrate) and the native ECD of an engineered Notch receptor would lead to high background levels of signaling.

Several recent studies have attempted to design fully genetically encodable synthetic systems to take advantage of the molecular mechanisms of the Delta/Notch signaling pathway while eliminating the limitations associated with the use of native ECDs of endogenous proteins. For example, TANGO is a method in which the interaction between a ligand and a receptor activates the intracellular viral protease TEV (tobacco etch virus) such that a membrane-anchored transcription factor is released (Barnea et al., 
A

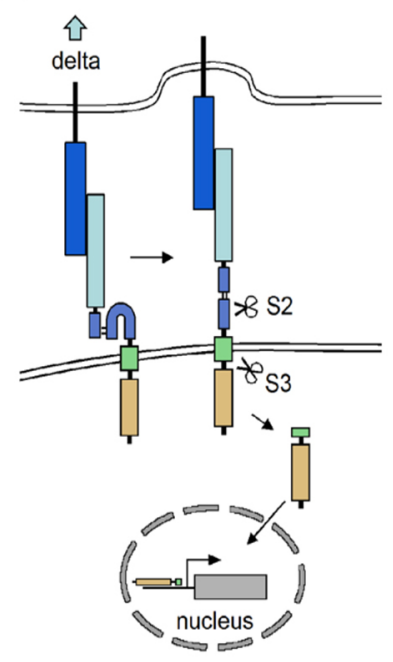

B

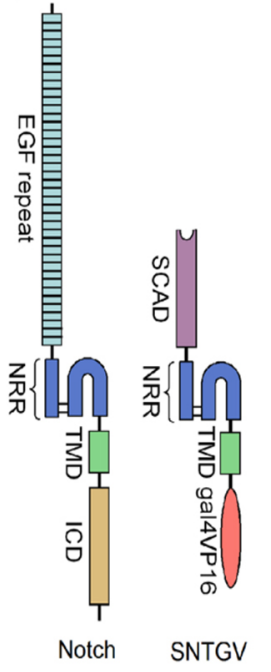

C

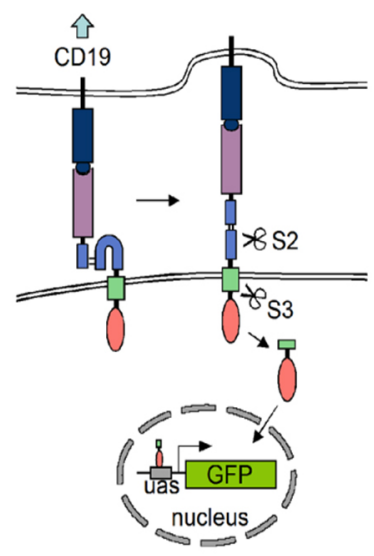

Fig. 1. A synthetic genetic system to record cell-cell contacts and manipulate interacting cells. (A) Molecular mechanism of Delta/Notch signaling. Upon ligand (Delta) binding, the NRR domain of the Notch receptor is partially unfolded, exposing the S2 site. Thereafter, it is sequentially cleaved, first in NRR ( 22 site) and then in the TMD (S3 site). After TMD cleavage, the ICD moves into the nucleus and activates transcription.

(B) Diagram depicting the domains of Notch and the engineered receptor, SNTGV. (C) SNTGV activation uses the molecular mechanism of the Delta/Notch signaling pathway. Upon CD19 binding, SNTGV is cleaved both in the NRR and TMD, and Gal4VP16 moves into the nucleus of the receiver cell to activate transcription of UASdependent genes, such as GFP in this example.
2008). A variation of the method called TANGO-TRACE has been developed to regulate gene expression in neurons in response to exposure to the neurotransmitters histamine and dopamine. The interaction of dopamine or histamine with TANGO receptors leads to a transient activation of a $\mathrm{G}$ protein that results in the labeling of the TANGO receptor-expressing neurons. One of the advantages of this system is that it only labels postsynaptic neurons when their synapses are activated, for example when a presynaptic neuron releases neurotransmitter in the synaptic cleft (Jagadish et al., 2014). Conversely, in its current implementation, TANGO-TRACE cannot be used to monitor cell-cell interactions based on physical contact. This feature would be advantageous to investigate cell-cell interactions that do not depend on the release of molecules, for example during development as migratory cells interact with their neighbors based on cell-cell adhesion. In addition, this feature would allow the investigation of neuronal connectivity in synaptic circuits without requiring knowledge of the neurotransmitters used by the connected neurons.

More recently, other variations of the Notch-Delta mechanism have been used to modify interacting cells genetically (Gordon et al., 2015; Morsut et al., 2016; Roybal et al., 2016). In this strategy, cells expressing an artificial ligand ('emitter' cells) activate a genetically modified Notch receptor on their interacting partners ('receiver' cells). Upon ligand-receptor interaction in sites of cellcell contact, the engineered receptor is cleaved in its transmembrane domain and releases a protein fragment that regulates transcription in the 'receiver' cell. This strategy has been used in vitro to investigate the mechanism of Notch/Delta signaling, to enable $\mathrm{T}$ cells to recognize tumors or to engineer cell interactions between cultured cells (Gordon et al., 2015; Morsut et al., 2016; Roybal et al., 2016). However, it remains to be shown whether ligandinduced intramembrane proteolysis can be used to monitor cell-cell interactions in vivo.

As an initial test, we have used the strategy of ligand-induced intramembrane proteolysis to investigate cell-cell interactions between glial cells and neurons in transgenic Drosophila in vivo. This method has allowed us to gain new information regarding neuron-glia interactions, and illustrates the potential of the method to perform experiments that are not possible with currently available methods. First, we demonstrate that the system can be used to activate gene expression in glial cells that contact specific subsets of neurons, using drivers that direct ligand expression into those neurons. This is an important feature as it allows the system to identify genetically (and eventually manipulate) cells in a selective manner even if no specific promoters exist for them. For example, we show that it is possible to label selectively a subset of glial cells that interact with olfactory neurons even though there are no specific drivers for these glial cells. Second, using this system we have observed that the distribution of glial cells can have a high degree of stereotypy. For example, we have observed that the astrocytes that interact with olfactory sensory neurons are preferentially localized in certain sectors of the antennal lobe. Third, we have observed that some of the astrocytes extend branches into two different functional areas of the brain (the antennal lobe and the subesophageal zone). This suggests that these particular astrocytes might bridge the function between these two brain areas, which is consistent with previous publications that imply a close functional relationship between these brain areas (Omoto et al., 2015). Based on these results, we anticipate that this synthetic genetic system will be useful for investigating cell-cell interactions during development in vivo, and has the potential to unveil wiring diagrams of neurons in brain circuits (Meinertzhagen and Lee, 2012; Lichtman and Denk, 2011).

\section{RESULTS}

\section{Monitoring cell-cell interactions in vitro}

To develop a robust system that would allow for the monitoring of cell-cell interactions in animals, we have engineered an artificial receptor called SCAD/NRR/TMD/GV, or SNTGV, for short. This design for the artificial Notch receptor is similar to those described recently (Gordon et al., 2015; Morsut et al., 2016). The SNTGV receptor retains the wild-type Notch NRR and TMD, but both the Notch ECD and ICD have been replaced by a single-chain antibody domain (SCAD) (Kochenderfer et al., 2009) and the transcriptional regulator Gal4VP16 (GV), respectively (Fig. 1B). The SCAD recognizes the $\mathrm{ECD}$ of murine $\mathrm{CD} 19$, thereby functioning as a receptor for this antigen. We have generated stable $\mathrm{CHO}$ cell lines carrying both the UAS-Histone2B-mcitrine (H2Bmcit) reporter (Sprinzak et al., 2010; Griesbeck et al., 2001; mcitrine is a YFP variant) and the SNTGV receptor (SNTGV/UAS-H2Bmcit cells) (Fig. 1C). In the absence of CD19 (the ligand), the SNTGV/UAS$\mathrm{H} 2 \mathrm{Bmcit}$ cells have negligible levels of $\mathrm{H} 2 \mathrm{Bmcit}$ expression (Fig. 2A; Fig. S1). By contrast, when SNTGV/UAS-H2Bmcit 
A co-culture with $\mathrm{CD}_{19}{ }^{+}$cells

$$
\text { Ohr }
$$

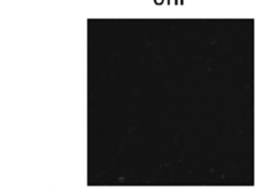

$12 \mathrm{hr}$

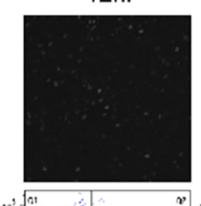

$24 \mathrm{hr}$

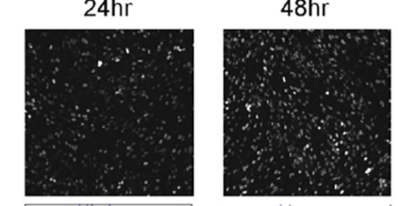

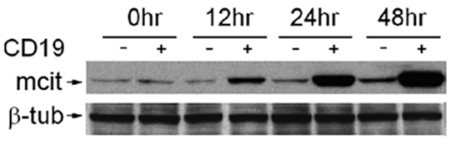

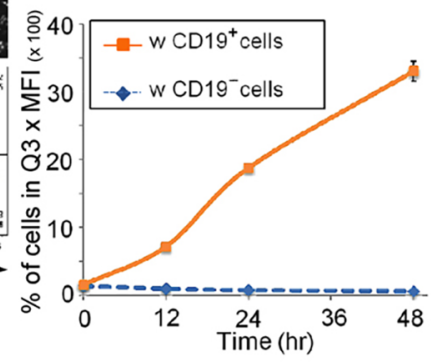

B

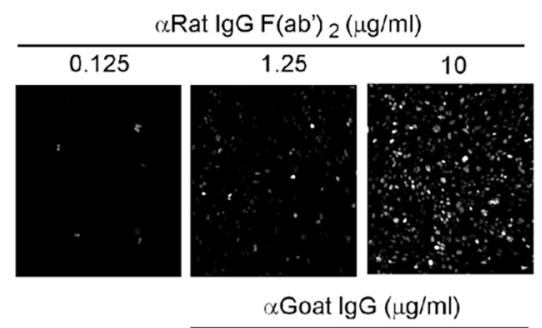

No $A b$.
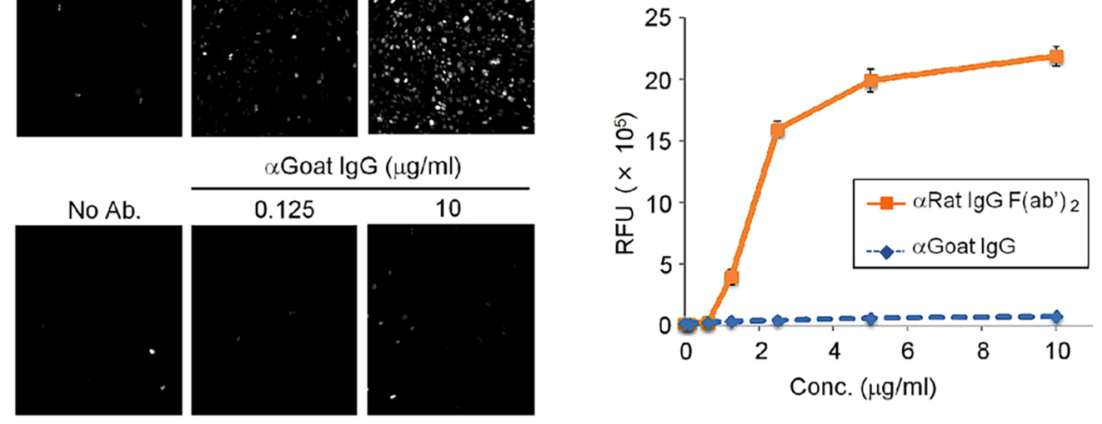

C
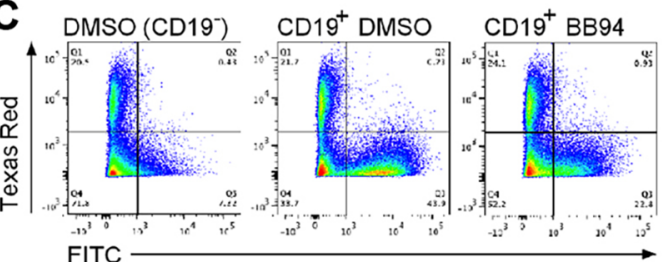
FITC CD19 ${ }^{+}$GM6001
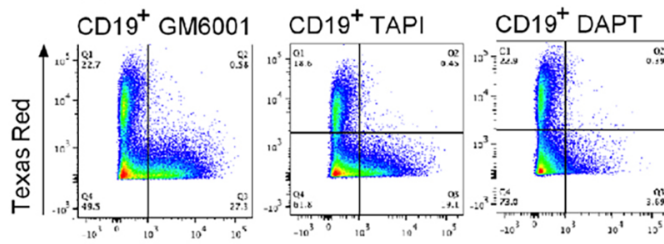

FITC
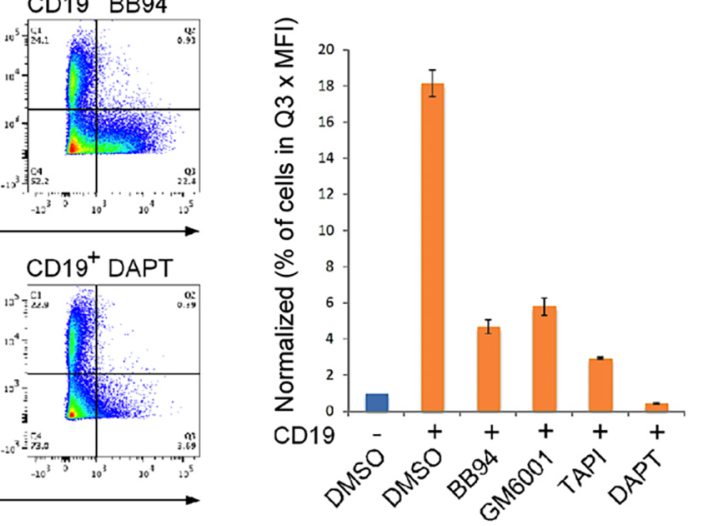

Fig. 2. Induction of reporter gene expression in vitro by cell-cell and cell-substrate interaction. (A) Induction of nuclear YFP expression [from a UAS-H2Bmcitrine (UAS-H2Bmcit) reporter cassette] at different time points after co-culturing SNTGV/UAS-H2Bmcit cells with $\mathrm{CD} 19^{+} / \mathrm{mCherry}^{+}$cells. Top left: microscopy images showing $\mathrm{H} 2 \mathrm{Bmcit}$ expression. Top right: western blot analysis of $\mathrm{H} 2 \mathrm{Bmcit}$ expression induced by co-culturing emitter and receiver cells. Bottom left: FACS plots showing the increase in H2Bmcit expression ( $x$-axis) in SNTGV/UAS-H2Bmcit cells in bottom right quadrant. The $y$-axis shows the intensity of red fluorescent protein in $\mathrm{CHO}$ cells. $\mathrm{CD} 19^{+} / \mathrm{mCherry}^{+}$emitter cells are located in the top left quadrant. Bottom right: quantification of the relative $\mathrm{H} 2 \mathrm{Bm}$ cit fluorescence intensity [bottom right quadrant; percentage of cells in Q3 (\%) multiplies mean FITC fluorescence intensity (MFI)] from the FACS analysis. Induction at 12 vs 0 h: 4.6 -fold $(P<0.0001)$; 48 vs 0 h=20.9-fold $(P<0.0001)$. (B) Immobilized ligand activates SNGTV signaling. Top left: anti-rat lgG $F\left(a b^{\prime}\right)_{2}$ antibody (which binds to SCAD) attached to plastic induces H2Bmcit expression in SNTGV/UAS-H2Bmcit cells. Bottom left: plastic-attached control antibody (which does not binds to SCAD) does not activate SNTGV signaling. Right: quantification of induction of H2Bmcit expression of SNGTV/UAS-H2Bmcit cells by immobilized anti-rat IgG F $\left(\mathrm{ab}^{\prime}\right) 2$ (relative fluorescence unit, RFU). Induction fold of anti-rat (positive ligand) versus anti-goat (negative control) antibodies: $1.25 \mu \mathrm{g} /$ $\mathrm{ml}=11.5$-fold $(P<0.005) ; 2.5 \mu \mathrm{g} / \mathrm{ml}=38$.3-fold $(P<0.0001) ; 5 \mu \mathrm{g} / \mathrm{ml}=34.6$-fold $(P<0.0001) ; 10 \mu \mathrm{g} / \mathrm{ml}=29.8$-fold $(P<0.0001)$. (C) The induction of SNGTV by its ligand (CD19) requires metalloprotease and gamma-secretase. Left: FACS analysis plots showing the effects of metalloprotease [batimastat (BB94), GM6001 and

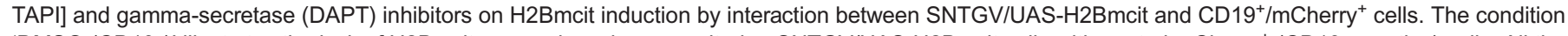
'DMSO (CD19-)' illustrates the lack of H2Bmcit expression when co-culturing SNTGV/UAS-H2Bmcit cells with control mCherry ${ }^{+}$(CD19-negative) cells. All the other conditions include SNTGV/UAS-H2Bmcit and CD19 $/ \mathrm{mCherry}^{+}$cells. Right: quantification of the relative fluorescence intensity from the FACS analysis shown on left. The values of the experimental conditions are normalized to the control 'DMSO (CD19-)'. Batimastat versus DMSO: $74.1 \%$ reduction $(P<0.0001)$; GM6001 versus DMSO: $67.7 \%$ reduction $(P<0.0001)$; TAPI versus DMSO: $84 \%$ reduction $(P<0.0001)$; DAPT versus DMSO: $98 \%$ reduction $(P<0.0001)$. 
cells are co-cultured with cells expressing CD19, there is robust induction of H2Bmcit expression (Fig. 2A; Fig. S1). H2Bmcit expression can be detected as early as $12 \mathrm{~h}$ after co-culture. After $48 \mathrm{~h}, \mathrm{H} 2 \mathrm{Bmcit}$ expression peaks at 30 -fold expression compared with uninduced controls (Fig. 2A). Importantly, co-culturing of SNTGV/UAS-H2Bmcit cells with $\mathrm{CHO}$ cells expressing rat Delta did not lead to $\mathrm{H} 2 \mathrm{Bmcit}$ expression (data not shown), indicating that the activation of SNTGV requires binding to its ligand, CD19. We also observed that this synthetic reporter system is activated in a concentration-dependent manner by an immobilized antibody that recognizes the ECD of SNTGV (anti-rat IgG) (Fig. 2B, top left). By contrast, no induction was observed when a non-specific antibody (anti-goat IgG) was used (Fig. 2B, bottom left). The system is highly sensitive, because we can observe robust $\mathrm{H} 2 \mathrm{Bm}$ cit expression with a concentration of the substrate-bound ligand as low as $1.25 \mu \mathrm{g} / \mathrm{ml}$, in the nanomolar range (Fig. 2B). This ligand concentration is similar to that previously reported for immobilized human Delta to activate Notch in the myoblast cell line C2 (Varnum-Finney et al., 2000).

It is hypothesized that the activation of Notch upon ligand binding involves a pulling force that exposes the NRR S2 site to metalloproteases (Nichols et al., 2007; Stephenson and Avis, 2012; Meloty-Kapella et al., 2012; Gordon et al., 2015). Consistent with previous works (Varnum-Finney et al., 2000), we have observed that SNTGV can be activated by ligands presented on the cell surface (Fig. 2A) or attached to a plastic substrate (Fig. 2B). However, we did not observe SNTGV activation when the antibody recognizing SCAD was applied in soluble form to the culture medium (data not shown). These results suggest that the mere binding of ligand (CD19) to the receptor (SNTGV) is not sufficient to expose the NRR S2 site. By contrast, our results are consistent with a model in which NRR acts as a mechanosensor that is partially unfolded by tension generated upon binding of an immobilized (cell- or substrate-bound) ligand to its receptor (Kopan and Ilagan, 2009; Stephenson and Avis, 2012; Meloty-Kapella et al., 2012; Gordon et al., 2015). In addition, incubating cells with the metalloprotease inhibitors batimastat, GM6001 and TAPI (which block the S2 site cleavage; Brou et al., 2000; Mumm et al., 2000), or with the gamma-secretase inhibitor DAPT (which blocks the S3 site cleavage; Brou et al., 2000; Mumm et al., 2000), reduced or eliminated, respectively, the induction of GFP expression observed by mixing the 'emitter' and 'receiver' cells (Fig. 2C). These observations suggest that the activation of SNTGV by CD19 binding probably recapitulates the S2 and S3 cleavages that occur when Delta and Notch interact, as recently described (Gordon et al., 2015).

\section{Monitoring glia-neuron interactions in the brain}

As an initial test of this system's ability to reveal cellular contacts in vivo, we explored the interactions between glial cells and neurons in the Drosophila nervous system. Glial cells are abundant in the nervous system, and many of their functions depend on the interactions between the glial and neuronal membranes. Interestingly, there are several different glial cell types in the Drosophila nervous system, including astrocytes, cortex glia, ensheathing glia, wrapping glia and subperineural glia. Each of these glial types has characteristic morphologies and functions, and interacts with neurons in different ways. For example, astrocytes have extensive membrane-membrane contacts with neurons as the highly branched astrocyte processes interact with synapses in the so-called 'tri-partite synapse' (Edwards and Meinertzhagen, 2010). By contrast, subperineural glia are thought to contribute to the blood-brain barrier, and only have limited contact with neurons
(Edwards and Meinertzhagen, 2010). Therefore, the variety of glial types provides a simple platform on which to test whether our system can reflect the different ways in which specific glial types interact with neurons.

To monitor contacts between neurons and glia in the Drosophila nervous system, we generated constructs tailored for expression in transgenic flies, namely a receptor called SNTG4 and CD19mch (see Materials and Methods for detailed description). To express the CD19mch ligand into specific glial types, we used the LexA/ LexAop bipartite expression system (del Valle Rodriguez et al., 2011; Venken et al., 2011), which allows for modular gene expression. We placed the CD19mch ligand under LexAopdependent control and used two different LexA drivers, alrmLexA::GAD or repo-LexA::GAD, to direct ligand expression into different glial types in Drosophila (Fig. 3). The alrm driver is strongly active in astrocytes and weak in most other glial cell types (Stork et al., 2012). The repo driver, on the other hand, is active in wrapping glia, subperineurial glia, perineurial glia and cortex glia, but weak in astrocytes (Freeman et al., 2003). Finally, we also included a UAS-GFP allele to report SNTG4 activation and combined these alleles by conventional genetic crosses (elavSNTG4; repo $>C D 19 m c h ; \quad U A S-G F P$ and elav-SNTG4; repo $>C D 19 m c h ; U A S-G F P)$. In the absence of CD19mch, there was low level ligand-independent background signal, with some weakly GFP-positive cells localized to the eye discs (Fig. 3D).

The different glial cell types interact with neurons different ways. Thus, we examined whether directing expression of CD19mch into specific sets of glial types with the repo and alrm drivers would lead to distinct patterns of reporter expression in neurons. The alrm promoter drove CD19mch expression in astrocytes throughout many regions of the late third instar larva nervous system, particularly in the central brain and the neuropils of the abdominal and thoracic neuromeres (Fig. 3B). GFP was induced in neurons throughout the nervous system in the same regions as those in which CD19mch was observed (Fig. 3B; Fig. S2A). The repo driver also led to $\mathrm{CD} 19 \mathrm{mch}$ expression throughout many regions of the nervous system, including the central brain, thoracic and abdominal neuromeres, and glial cells that wrap the peripheral nerves (red fibers in Fig. 3C and Fig. S2B). This pattern of ligand expression led to $\mathrm{GFP}^{+}$neurons in the same or adjacent areas to where CD19mch was observed. No GFP expression was observed in any of these areas in the absence of the LexA driver for the ligand (Fig. 3D) or the SNTG4 receptor (data not shown). These data indicate that the GFP signal observed upon co-expressing CD19mch and the SNTG4 receptor is based on the physical interaction between neurons and glia.

The GFP expression pattern induced by repo-driven ligand overlaps with that of alrm-driven ligand in certain areas of the nervous system (namely the mushroom body and the neuropils of the thoracic and abdominal neuromeres). However, as expected given the different types of glial cells targeted by the repo and alrm drivers (Stork et al., 2012), there were also some differences between the regions in which GFP was induced in neurons when the ligand was directed by alrm and repo (Fig. 3; Fig. S2). For example, in the optic lobe the GFP induction in neurons was very strong with the repo, but very weak with the alrm driver (Figs 3 and 4). This observation is consistent with the robust expression of $\mathrm{CD} 19 \mathrm{mch}$ in the optic lobe with the repo driver, but very weak expression here with the alrm driver (Figs 3 and 4). These data show that expressing ligand in discrete subpopulations of glia can reveal different cell-cell interactions, highlighting the specificity and versatility of the system. However, the repo and alrm drivers 
A

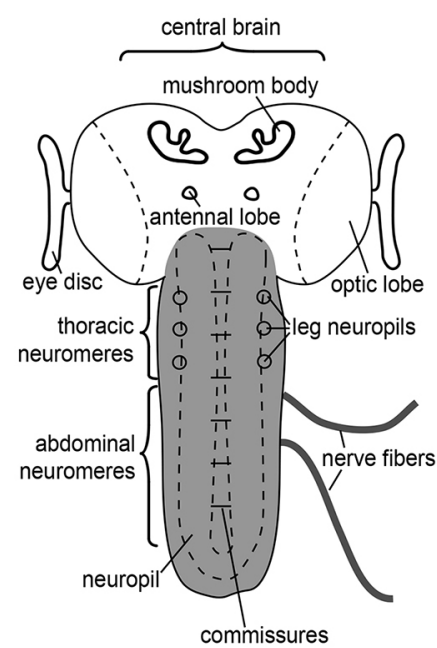

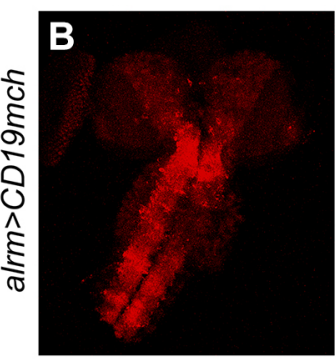
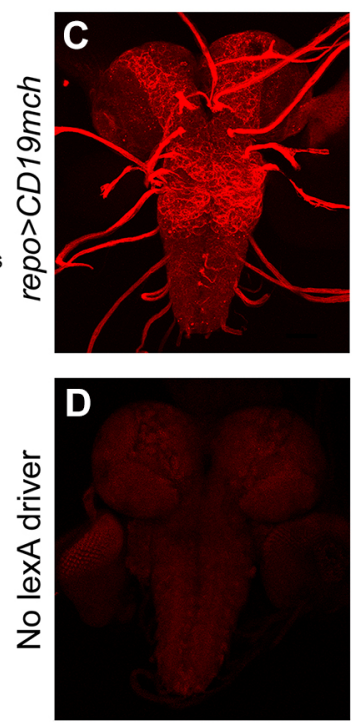
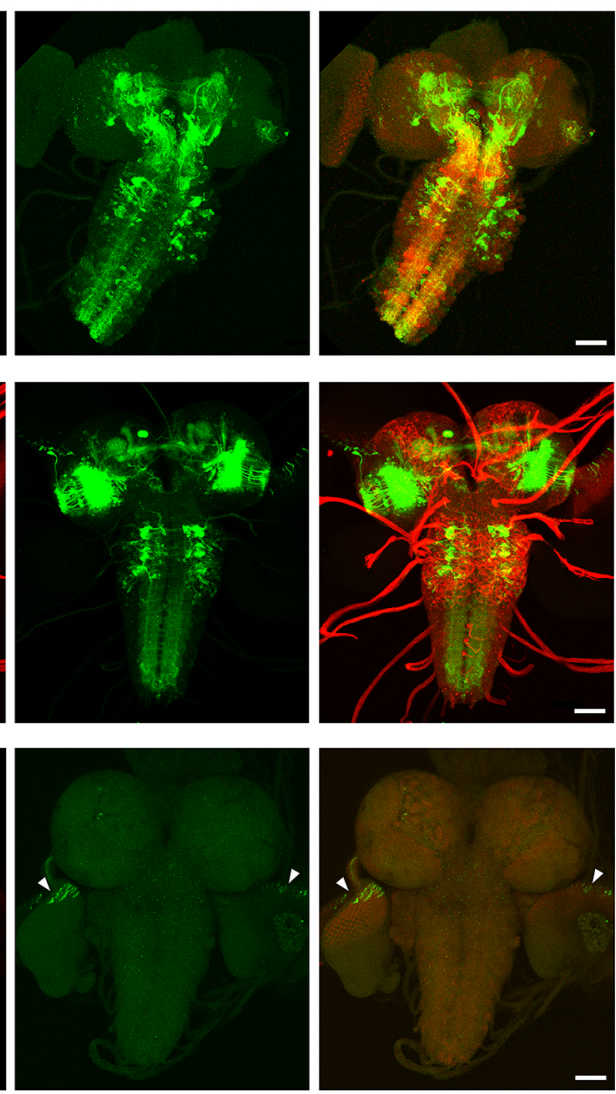

Fig. 3. Monitoring glia-neuron contacts in the Drosophila nervous system. (A) Diagram of the Drosophila larval nervous system indicating the main regions and structures in the brain and ventral nerve cord (shadowed in gray). (B,C) Expression of the CD19mch ligand by the alrm (B) and repo (C) drivers leads to GFP expression in elav-SNTG4 neurons throughout the larval nervous system. (D) Control flies without the LexA driver have weak GFP background expression (ligand independent) in the larval eye discs (arrowheads). For all panels, left shows distribution of CD19mch ${ }^{+}$emitter cells; middle shows distribution of GFP ${ }^{+}$neurons; right shows merged imaged of CD19mch (red), GFP (green). Scale bars: $50 \mu \mathrm{m}$.

directed expression of the ligand in glial cells broadly distributed throughout the nervous system. Consequently, we observed broad activation of GFP in a large number of neurons throughout the brain and ventral nerve cord, as there was no regional specificity for either the emitter or receiver cells. This made it difficult to study in detail or quantify the interactions between neurons and glia. To overcome this limitation, we attempted to investigate the interactions between highly specific subsets of neurons and the glia that contact them.

\section{Monitoring interactions between glia and specific types of neurons}

Understanding the development and function of the brain will require the ability to monitor, and eventually modify, the interactions between neurons and glia. For example, many recent studies have shown that astrocytes play important roles in synaptic formation and in modulating the functions of neural circuits and behavior in Drosophila (Zwarts et al., 2015; Freeman, 2015) However, as shown in Fig. 3, the interactions between glia and neurons are ubiquitous throughout the nervous system. Glial promoters such as alrm and repo will direct transgene expression into glial cells in all areas of the nervous system, and, consequently, these glial cells will interact with widely distributed neurons. Thus, any genetic manipulation that depends on general promoters such as alrm or repo would be difficult to interpret, because they would affect the interaction between neurons and glia in a global manner. For many experiments it would be necessary to selectively target populations of glial cells located in discrete areas of the nervous system in order to achieve highly specific manipulations. Interestingly, there are many neuron-specific promoters (in Drosophila, zebrafish or mice) that can be used to direct transgene expression into selected brain areas. For example, in Drosophila there are promoters or drivers than can be used to express transgenes selectively in projection neurons in the antennal lobe (Stocker et al., 1997), or specific photoreceptors in the compound eyes (Bowtell et al., 1991). Similarly, in mammals, there are promoters to direct expression of transgenes in mitral cells of the olfactory bulb (Nagai et al., 2005) or Purkinje neurons in the cerebellum (Oberdick et al., 1990). By contrast, currently there are no known promoters that can be used to direct transgene expression selectively in glial cells located in discrete areas of the nervous system. Therefore, to manipulate the astrocytes that contact subsets of neurons in discrete areas of the Drosophila nervous system, we utilized highly specific drivers that direct expression of the CD19 ligand into specific neuronal types, whereas the SNTG4 receptor was expressed in astrocytes driven by alrm promoter. We used three different drivers to direct ligand expression into specific subsets of neurons: (1) MB247-LexA::VP16 for Kenyon cells (KCs) of the mushroom body, (2) Orco-LexA::VP16 for olfactory sensory neurons (OSNs), and (3) Pdf-LexA for Pigment-dispersing factor (PDF)-positive neurons in ventral nerve cord and central brain. We analyzed the contacts between these selective subsets of neurons and the glia with which they interact during the wandering larval stage, and we observed that selective subsets of astrocytes were 

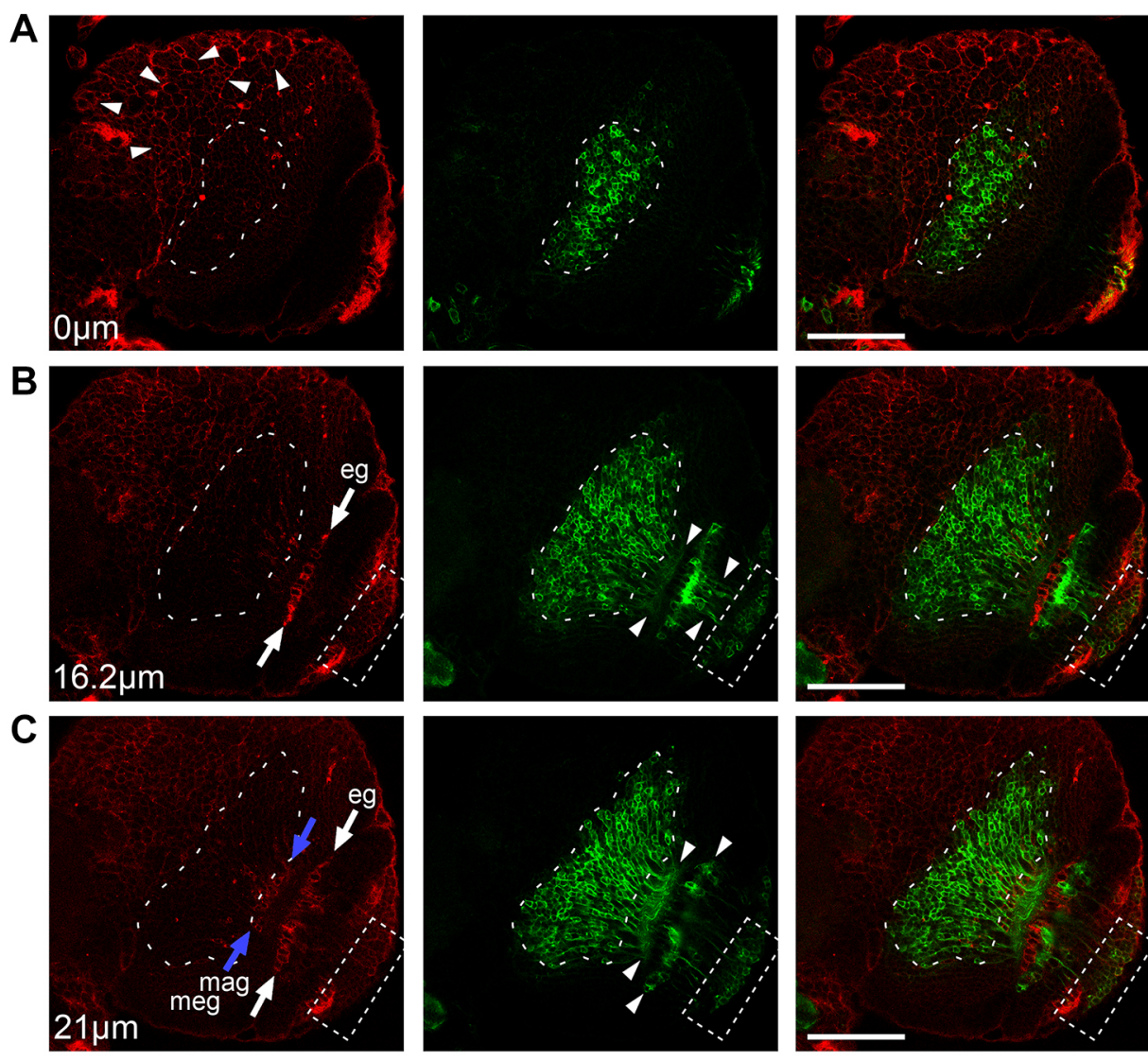

Fig. 4. Differential induction of GFP in the central brain and optic lobe by expression of CD19mch ligand by a repo driver. (A-C) GFP expression was induced in the neurons of larval optic lobe (dashed oval and rectangular outlines) when CD19mch was expressed in larval optic lobe glia cells $(B, C$, white and blue arrows) by the repo driver. By contrast, CD19mch expression in the central brain (A, white arrowheads) did not induce GFP expression in neurons in those areas. Serial single optical sections (A: $z=0 \mu \mathrm{m} ; \mathrm{B}$ : $z=16.2 \mu \mathrm{m} ; C: z=21 \mu \mathrm{m}$ ) of larval optic lobe from confocal images show the distribution of

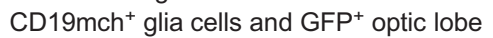
neurons. (A) The cell bodies of medulla neurons express GFP strongly and form a cluster (outlined), with sparse, weakly expressing $\mathrm{CD} 19 \mathrm{mch}^{+}$glial cells inside the cluster. (B) The cell bodies of lamina neurons are also $\mathrm{GFP}^{+}$and form a second cluster (boxed) with weakly expressing CD19mch ${ }^{+}$glial cells present inside the cluster. The neurites of $\mathrm{GFP}^{+}$lamina and medulla neurons (arrowheads) make contact with a row of epithelial glia (e.g. white arrows) that express high levels of CD19mch. (C) The neurites of $\mathrm{GFP}^{+}$neurons (arrowheads) interact with a second row of strongly expressing

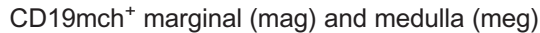
glia (blue arrows). For all panels, left shows distribution of CD19mch ${ }^{+}$emitter cells; middle shows $\mathrm{GFP}^{+}$neurons; right shows merged imaged of CD19mch (red), GFP (green). Scale bars: $20 \mu \mathrm{m}$. induced to express GFP in different CNS regions, as expected from the position of the ligand-expressing neurons (Figs 5-7).

First, when mCD19mch was expressed in central brain neurons (including KCs) by the MB247 driver, more than 20 astrocytes were labeled in the central brain surrounding the mushroom body, the antennal lobe and subesophageal zone (Fig. 5A) in wandering larvae. We observed that $\mathrm{GFP}^{+}$astrocytes near the mushroom bodies infiltrated their processes into the calyx and lobes of mushroom bodies (Fig. 5B). In addition, we noticed that the MB247 driver also directed CD19 expression in other neurons outside the mushroom bodies (Fig. 5C, arrows and arrowheads), and accordingly, there was GFP induction in astrocytes at the antennal lobe and subesophageal zone of the MB247 animals (Fig. 5D, arrow).

When CD19mch was expressed in OSNs, we observed selective GFP expression in astrocytes near the antennal lobes (Fig. 6C). The cell bodies of these $\mathrm{GFP}^{+}$astrocytes contacting with OSN axon terminals were most commonly located in the medial and ventrolateral regions of the antennal lobes, but never in the dorsolateral regions (Fig. 7B). Interestingly, some of those $\mathrm{GFP}^{+}$ astrocytes infiltrate not only the antennal lobes, but also the dorsal part of the subesophageal zone (Fig. 6C, arrowhead in middle panel), consistent with previous findings of astrocytes in larval central brain (Omoto et al., 2015). Moreover, we found that the cell body size of the $\mathrm{GFP}^{+}$astrocytes surrounding the mushroom bodies (induced by the MB247 driver) is larger than that of the astrocytes close to the antennal lobes (induced by the Orco driver). By measuring the area of the largest optical cross-section of the $\mathrm{GFP}^{+}$ astrocyte cell bodies, we showed that the astrocytes labeled near the mushroom bodies of MB247 animals are 1.4-fold larger than the antennal lobe astrocytes in Orco animals (Fig. 7C) [Orco: $96.08 \pm 25.90 \mu \mathrm{m}^{2}$ (mean \pm s.d.), $n=35$ from 10 animals; MB247:
$135.71 \pm 32.0 \mu \mathrm{m}^{2}, n=64$ from 6 animals; $t$-test, $\left.P<0.0001\right]$. This observation indicates that astrocytes in different brain areas of the Drosophila nervous system may be functionally heterogeneous.

When CD19mch was expressed under the Pdf-LexA driver, we observed GFP induction in astrocytes near the larval optic lobe (Fig. 6Da, white arrow in right panel) and in the distal end of the ventral nerve cord (Fig. 6Db, white arrows in right panel). This distribution of $\mathrm{GFP}^{+}$astrocytes is consistent with the location of the two groups of $\mathrm{PDF}^{+}$neurons in larval nervous system: (1) lateral neurons in the central brain close to the optic lobe (Fig. 6Da, blue arrow in middle panel), which receive input from photoreceptors (Sprecher et al., 2011), and (2) motor neurons in the last two segments of ventral nerve cord (Fig. 6Db, blue arrows in middle panel) that innervate the hindgut to control muscle contraction (Helfrich-Forster, 1997; Talsma et al., 2012; Zhang et al., 2014).

To evaluate the consistency of the system, we counted the number of the GFP-labeled astrocytes in the ventral nerve cord terminal of $P d f$ animals and the antennal lobes of Orco animals, where it was possible to count the number of $\mathrm{GFP}^{+}$cells accurately. Because the number of $\mathrm{GFP}^{+}$astrocytes in $M B 247$ animals was very high $(>20)$, and their processes were very dense, it was very challenging to identify individual cells unambiguously in order to count them. Similarly, the induction of GFP in astrocytes in the central brain of animals with ligand expressed with the $P d f$ driver was weak, and this made quantification difficult. The GFP induction patterns in the antennal lobes of Orco driver animals and ventral nerve cord of $P d f$ driver animals are highly consistent among individuals (Fig. 7A). There were between five and eight (mean \pm s.d.: $6.57 \pm 0.87, n=21$ ) astrocytes labeled in the ventral nerve cord terminal of $P d f$ animals, and between one and four (mean \pm s.d.:1.68 $\pm 0.74, n=25$ ) astrocytes in the antennal lobes of Orco animals. These experiments 
A

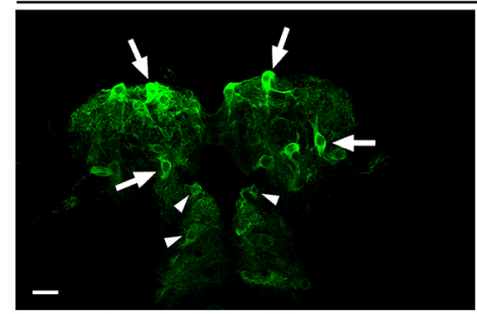

MB247>CD19mch

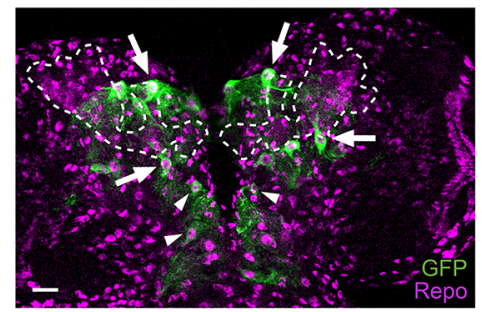

B

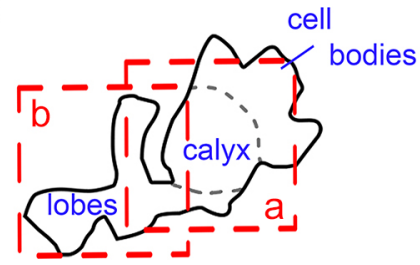

MB247>CD19mch

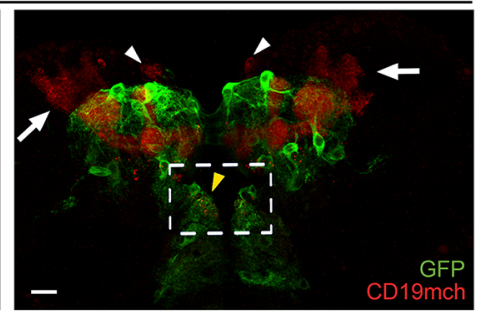

No LexA driver

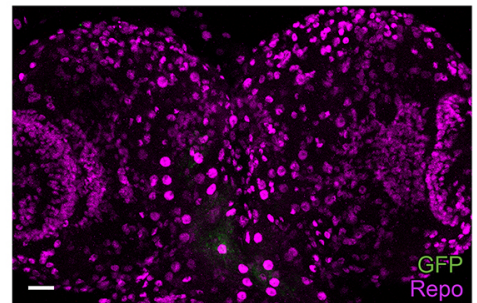

C

MB247>CD19mch

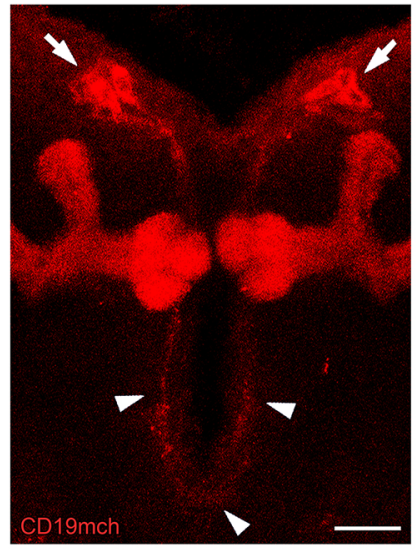

D

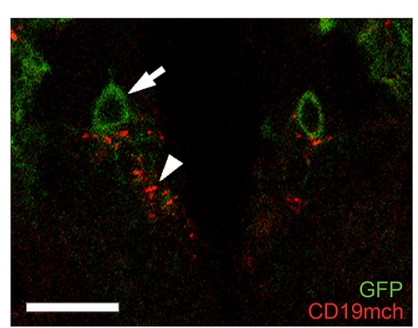

Fig. 5. Monitoring neuron-astrocyte contacts selectively in Drosophila larval mushroom bodies. (A) Induction of GFP in central brain astrocytes (arrows and arrowheads in top left panel) triggered by CD19mch expressed under the MB247 driver (red signals in top right panel). SNTG4 receptor was expressed in all astrocytes using the alrm promoter. The majority of the CD19mch ${ }^{+}$neurons driven by MB247 are Kenyon cells (KCs) in mushroom bodies (arrows in top right panel), and are located next to GFP ${ }^{+}$astrocytes (arrows in top left panel). In addition to KCs, the MB247 driver also expresses CD19mch in other neurons outside of the mushroom body (cell bodies indicated by white arrowheads and neurites by yellow arrowhead in top right panel), which leads to GFP expression in astrocytes located in the subesophageal zone and antennal lobe (arrowheads in top left). The enlarged image of the boxed region is shown in D. Bottom left: immunostaining against Repo shows the location of all glial cell nuclei. The mushroom bodies are outlined. Bottom right: control larva without the LexA driver has no GFP expression. (B) Scheme showing the three domains of the right mushroom body shown in A: KC cell bodies are located in the top right corner. Dendrites from KCs branch in the calyx, and their axons project to the lobes. Single optical sections of the calyx and dorsal lobe are shown in Ba and Bb, respectively. In Ba and $\mathrm{Bb}$, the branches of the GFP-positive astrocytes surround the mushroom body. The arrows indicate the dendrites [branching in the calyx (Ba)] and axons [branching in the dorsal lobe (Bb)] of CD19mch ${ }^{+} \mathrm{KCs}$. (C) CD19mch expression pattern driven by MB247. In addition to the KCs in the mushroom bodies,

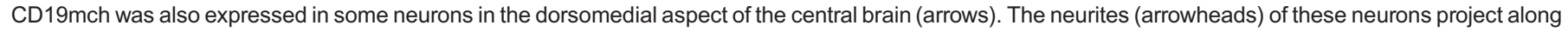

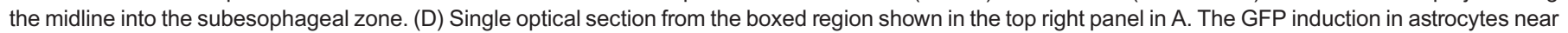

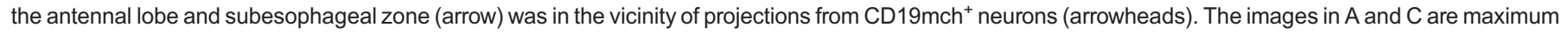
projections of confocal microscopy images. Scale bars: $20 \mu \mathrm{m}$.

demonstrate that directing expression of the ligand into a subset of neurons localized to a restricted area of the nervous system activates transcription in a very selective subset of astrocytes that make contact with those neurons in a reliable manner.

\section{DISCUSSION}

Our experiments demonstrate that it is possible to take advantage of the molecular mechanisms of the Notch pathway to detect cellcell interactions in vivo. We have generated transgenic animals in which cells expressing an artificial ligand ('emitter' cells) activate a genetically modified Notch receptor on their interacting partners ('receiver' cells). Using this system, we have shown that expressing the ligand in glial cells activates transcription in neurons throughout the Drosophila brain and ventral nerve cord. For these experiments, we used the repo driver, which is highly active in subperineurial, perineurial, cortex and ensheathing glia, but weak in astrocytes, and the alrm driver, which is active in astrocytes, but much weaker in other glial types (Freeman et al., 2003). As expected, the set of neurons activated is different when the ligand is expressed under the repo or alrm drivers. These different patterns of GFP induction are likely to be due to several factors. First, alrm and repo drivers are active in different brain regions. For instance, strong ligand expression can be detected in the optic lobe with the repo (Fig. 3C; Fig. 4) but not the alrm (Fig. 3B) driver. Consequently, the GFP induction is robust in the optic lobe of repo (Fig. 3C; Fig. 4) but not alrm (Fig. 3B) animals. Second, even within the same region, the repo and alrm drivers are active in different populations of glial cells (Stork et al., 2012). In particular, the alrm driver is active in astrocytes (Freeman et al., 2003), which have a large membrane surface because they have highly branched processes that occupy a large fraction of the neuropil. The contact area between neurons and astrocytes is large because their interaction occurs between highly ramified processes from neurons (dendrites and axonal arborizations) and astrocytes (astrocyte processes) (Stork et al., 2012). Consistent with this observation, although the overall expression of CD19mch in the central brain with the alrm driver (Fig. 3B; Fig. S2) was weaker than with the repo driver (Fig. 3C; Fig. S2), the expression of CD19mch in astrocytes with the alrm driver may account for the robust GFP induction in mushroom body, antennal lobe and subesophageal zone neurons (Fig. 3B; Fig. S2). Third, it is possible that not all areas of the cell membrane that participate in 
A

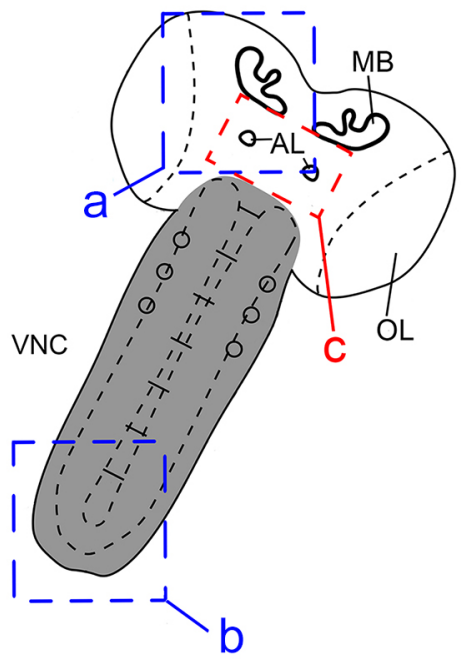

B
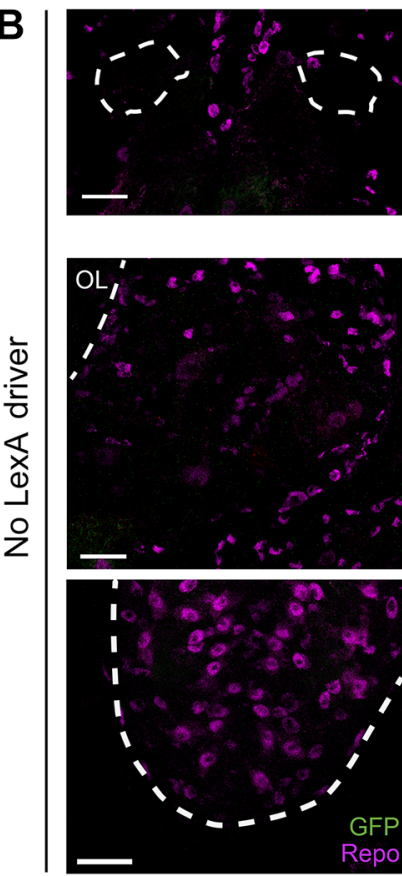

C

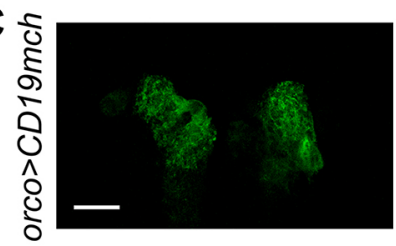

D

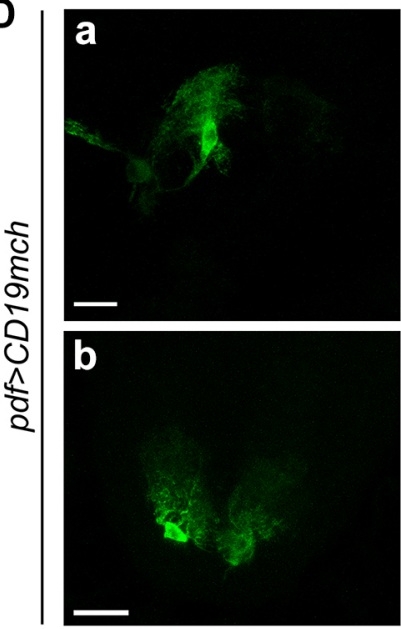

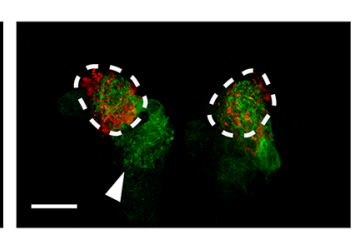
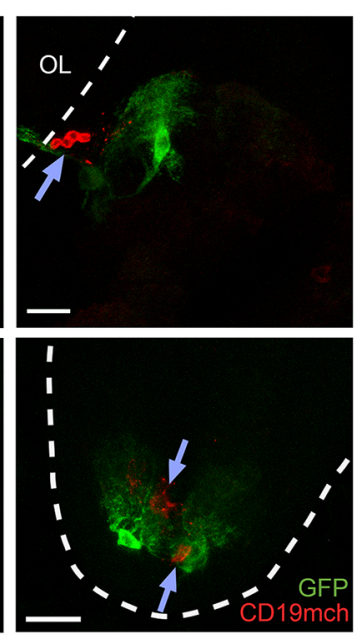
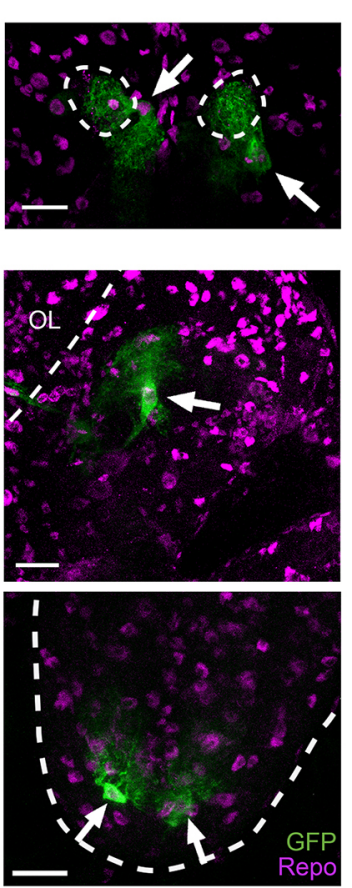

Fig. 6. Monitoring neuron-astrocyte contacts selectively in larval antennal lobe, central brain and ventral nerve cord. (A) Diagram of the Drosophila larval nervous system indicating the regions shown in $B$ $D$. The region in the red rectangle including antennal lobe $(A L)$ is shown in $C$ and in the top panel of $B$. The region in the top blue square in the central brain is shown in $\mathrm{Da}$ and in the middle panel of $\mathrm{B}$. The distal part of the ventral nerve cord (VNC) in the bottom blue square is shown in $\mathrm{Db}$ and in the bottom panel of $\mathrm{B}$. (B) Control larva without the LexA driver has no GFP expression in the antennal lobes (top, outlined), central brain (middle) or the distal part of VNC (bottom, outlined). (C) The Orco driver induces CD19mch expression in most OSNs. CD19 $\mathrm{mch}^{+}$axons from OSNs projecting into the antennal lobe (red in the outlined regions) induced GFP expression in antennal lobe astrocytes (white arrows in the right panel). The $\mathrm{GFP}^{+}$astrocytes located in the medial sector of the larval antennal lobes also infiltrate into the subesophageal zone (white arrowhead in middle panel). (D) GFP induction in selective astrocytes in the central brain (Da) and ventral nerve cord $(\mathrm{Db})$ when $P d f$ driver directed CD19mch expression into PDF neurons. In $\mathrm{Da}$, the dashed lines mark the boundaries between the larval optic lobe (OL) and central brain, and in Db the dashed line contours the ventral nerve cord. The CD19mch ${ }^{+}$PDF neurons (blue arrows in middle panels) induce GFP expression in a small set of astrocytes in their vicinity (white arrows in right panels). All images in this figure are maximum projections of confocal microscopy stacks. MB, mushroom body. Scale bars: $20 \mu \mathrm{m}$. the interaction between neurons and glia have the same ability to activate the receptor. For example, in the optic lobe with the repo driver there is strong GFP induction in the medulla and lamina neurons, but the CD19mch signal close to the cell bodies of $\mathrm{GFP}^{+}$ neurons is very weak, or absent (Fig. 4). By contrast, there is clear contact between the neurites of the $\mathrm{GFP}^{+}$neurons and $\mathrm{CD} 19 \mathrm{mch}^{+}$ glial cells clustered in two bands in the optic lobe, the marginal and medulla glia, and the epithelial glia (Fig. 4B,C). As a counterexample, in that same image there is strong CD19mch expression in glial cells in the central brain in areas where there are many neuronal cell bodies, but there is no GFP induction in neurons in those areas (Fig. 4A). Interestingly, those central brain areas with no $\mathrm{GFP}^{+}$neuronal induction also contain cortex glia, which primarily make contact with the cell bodies of neurons, and the repo driver is expected to direct ligand expression into these cortex glia. These observations suggest that the interaction between neurites and glial processes may be more effective at activating the receptor than interactions between cell bodies. This interpretation is also consistent with the observation of strong GFP induction in central brain neurons when the ligand is expressed in astrocytes (with the alrm driver), as astrocyte processes interact with neuronal processes in synapses (Figs 3, 5-7; Fig. S2). Finally, although our data indicate that the ligand CD19mch is present throughout the neuronal membrane, including cell bodies, axons and dendrites, its distribution may not be strictly uniform along the plasma membrane (Fig. 5C). Varying density of the ligand in the plasma membrane could account for the ability to detect some neuron-glia interactions, but not others. 
A
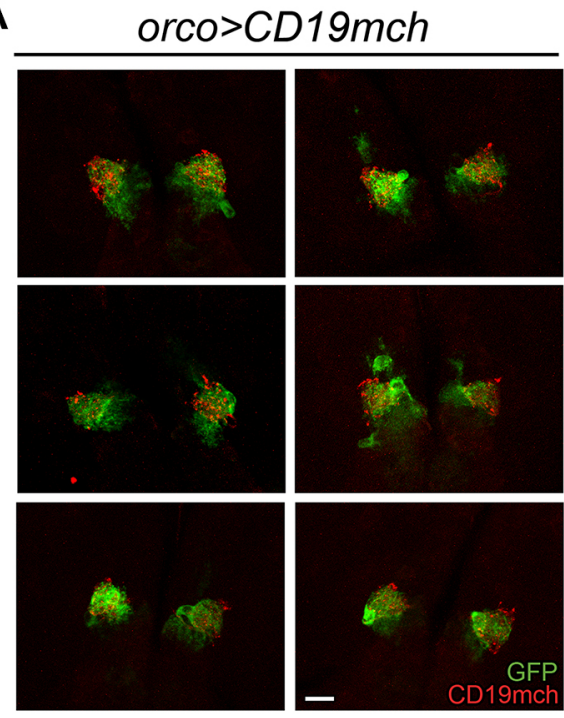

B
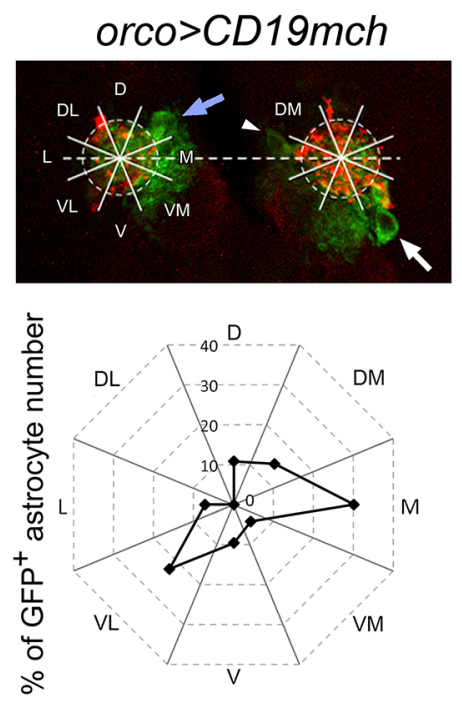

\section{C}

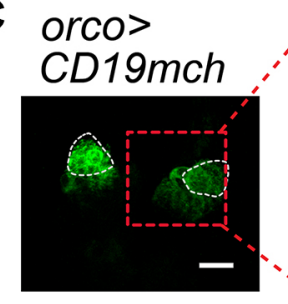

MB247>
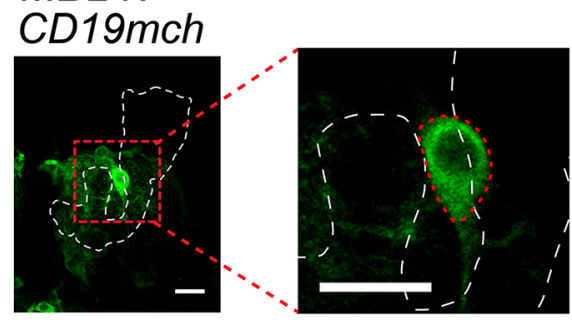

Fig. 7. Distribution and characteristics of astrocytes interacting with ligand-expressing neurons. (A) Examples of the GFP expression patterns induced in astrocytes in the antennal lobe (with ligand expressed in OSNs with Orco driver; left two panels) and ventral nerve cord (with ligand expressed by Pdf driver; right two panels) show highly similar distribution of induced astrocytes and GFP intensity between hemispheres and animals. Maximum projections of confocal microscopy images. (B) The top panel shows an example illustrating how the locations of astrocyte cell bodies were determined in the antennal lobe. The two antennal lobes from each animal were aligned to generate the horizon line. Each antennal lobe was equally divided into eight sectors: dorsal (D), dorsolateral (DL), lateral (L), ventrolateral $(\mathrm{VL})$, ventral $(\mathrm{V})$, ventromedial (VM), medial $(\mathrm{M})$ and dorsomedial (DM). The sectors where the cell bodies of the GFP ${ }^{+}$astrocytes located were recorded. In this example, one astrocyte cell body at the left antennal lobe is in the DM sector (blue arrow); two astrocyte cell bodies at the right antennal lobe are in the M (white arrowhead) and VL (white arrow) sectors. The bottom panel shows the percentage of the astrocytes located in the different sectors. The GFP ${ }^{+}$astrocytes in the antennal lobes are most commonly located in the M (29.8\%) and VL $(22.6 \%)$ sectors, but no astrocytes were located in the DL sector (total 84 astrocytes from 25 larval brains). (C) Size of cell bodies of GFP ${ }^{+}$astrocytes in the antennal lobe and mushroom body. Exemplars of two astrocyte cell bodies (red dashed outlines) in the antennal lobe (top, outlined in white) of an Orco animal and in the mushroom body (bottom, outlined in white) of an MB247 animal. Left panels show the maximum projection images of each GFP ${ }^{+}$ astrocyte; right panels show the enlarged single optical sections, where the area size of cell bodies (red dashed outlines) were measured. Scale bars: $20 \mu \mathrm{m}$.
Furthermore, we demonstrate that directing expression of the ligand into a subset of neurons localized to a restricted area of the nervous system activates transcription in a very selective subset of astrocytes that make contact with those neurons. We were able to activate transcription selectively in specific subsets of astrocytes located in the following regions: (1) antennal lobe (with a neuronal driver for OSNs), (2) mushroom body (with a neuronal driver for $\mathrm{KCs}$ ), and (3) central brain regions next to the optic lobe and terminal end of the ventral nerve cord (with a driver for PDF neurons). This observation indicates that even if there are no specific promoters capable of directly driving expression of transgenes into certain cell types (such as antennal lobe or mushroom body astrocytes), this strategy makes it possible to genetically manipulate highly specific populations of cells based not on the genes that they express, but on the cells with which they interact.

This system has allowed us to gain new insights into the interactions between neurons and glia. We have shown that the distribution of glial cells can have a high degree of stereotypy. For example, we have observed that the astrocytes that interact with olfactory sensory neurons are preferentially localized in certain sectors of the antennal lobe, but are rarely, if ever, present in other sectors (Fig. 7B). We have also observed that the astrocytes surrounding the ventral sectors of the antennal lobe also extend branches into the subesophageal zone. This suggests that this particular astrocyte might bridge the function between the antennal lobe and the subesophageal zone, consistent with previous publications that imply a close functional relationship between these two brain areas in the larval brain (Omoto et al., 2015). Finally, we have observed that the size of the cell bodies of astrocytes that branch in the mushroom body are larger than those that branch in the antennal lobe. This observation suggests that there may exist an unexplored degree of functional specificity for Drosophila glial cells that are located in different brain areas.

There are many advantages of signaling systems based on ligandinduced membrane proteolysis. (1) They are fully genetically encoded, and therefore could be used with high reproducibility in transgenic animals. Moreover, as we demonstrate here, the ligand and/or the receptor could be driven with promoters specific to selective cell populations to enable monitoring of cell-cell interactions from specific cell types and developmental stages. Importantly, we have confirmed that in cells that express both the ligand and the receptor, on the same membrane in cis, there is no activation of the reporter (Fig. S3). This observation indicates that in situations where a cell expresses both the receptor and the ligand, the 
activation of the reporter will indicate a trans-activation, between interacting neighboring cells. (2) They can be used in any species in which transgenesis is possible. This is particularly important for mice (Anderson and Ingham, 2003), Drosophila (Bellen et al., 2010) and zebrafish (Fetcho and Liu, 1998), three model organisms with extremely powerful genetics that are of great interest to developmental biologists and neurobiologists. (3) The interacting cells can be studied both in vivo (combined with live imaging, electrophysiological recordings and optical monitoring of activity) or in fixed tissue (by fluorescent or electron microscopy). (4) The system can be used in high-throughput experiments because, unlike electron microscopy, it is not labor intensive. In addition, as we have shown here, it is reproducible between animals because it does not require injections of the signaling components or any other chemical. (5) The system can be used to modify the interacting cells genetically. For instance, it could be used to induce the expression of transgenes such as genetically encoded calcium sensors (Tian et al., 2012) or optogenetic constructs (Yizhar et al., 2011). (6) Lastly, it could be used to control cell fate or function by regulating endogenous genes indirectly through nuclear translocation of drivers such as Cre, Flp, LexA or TetA (Lewandoski, 2001; Venken et al., 2011; del Valle Rodriguez et al., 2011), or directly by fusing endogenous transcription factors to the artificial receptor. We anticipate that these synthetic genetic systems will be particularly useful to investigate cell-cell interactions during development in vivo.

Finally, there is an urgent need for new methods to map synaptic connectivity in neural circuits (Meinertzhagen and Lee, 2012; Lichtman and Denk, 2011). It is generally agreed that identifying how neurons in those circuits are connected to one another is crucial to understanding the computations that take place in brain circuits. In addition, recent research indicates that aberrant neuronal wiring might be the cause of several neurodevelopmental disorders, further emphasizing the importance of identifying the wiring map of brain circuits (Peca and Feng, 2012). In its current implementation, the system that we have described cannot be used to trace brain circuits. In order to use the described system to study neuronal connectivity it would be necessary to localize the receptor or ligand specifically into presynaptic and postsynaptic sites (Sudhof, 2012; Sheng, 2001). Our lab is currently working to selectively target the ligand and receptor to synaptic sites in order to optimize the system for identifying synaptic connections. Synaptic localization will also eliminate the possibility of reporter activation between neurons whose membranes are close to each other (for instance, between fasciculating axons) but are not connected by synapses. In this study, we have shown that this system can be used to analyze neuron-glia interactions in vivo. We anticipate that with further optimization, control of transcription via ligand-induced intramembrane proteolysis has the potential to be used to investigate cell-cell interactions during development in vivo, and elucidate the wiring map of neuronal circuits.

\section{MATERIALS AND METHODS \\ Generation of genetic constructs and production of viral particles}

SNTGV was constructed by fusing a single chain antibody (SCAD) that recognizes the mouse CD19, the NRR and TMD from human notch 1, and Ga14VP16. The SCAD included amino acids 1-289 from the monoclonal 1D3-28z.1-3 (Kochenderfer et al., 2009). The NRR domain and TMD comprised amino acid 1446-1880 of human notch 1. Gal4VP16 was then fused after the notch $1 \mathrm{TMD}$, and the entire SNTGV was subcloned into the FUW lentiviral backbone (Lois et al., 2002). Lentiviral particles encoding
SNTGV were generated as previously described (Lois et al., 2002). For generating retroviral particles expressing mouse CD19, the MSGV-CD19 plasmid (Kochenderfer et al., 2009) was co-transfected with pCL-Eco and VSVg as previously described (Lin et al., 2010). The sequences of SNTGV and CD19 are available in the supplementary Materials and Methods.

\section{Generation of stable cell lines}

The UAS-H2B-citrine reporter $\mathrm{CHO}$ cell line was kindly provided by $\mathrm{Dr}$ Elowitz (Caltech). Citrine is a variant of YFP, and we will refer to this reporter as UAS-H2Bmcit. UAS-H2Bmcit cells were grown as described previously (Sprinzak et al., 2010). To stably express SNTGV, UASH2Bmcit cells were infected with a SNTGV lentivirus. SNTGV/UASH2Bmcit cells were sorted into single cells in a 96-well plate to generate clonal cell lines. Clones with low fluorescence background and high inducibility were chosen for further experiments. To generate stable lines of control emitter cells, CHO cells were first infected by retrovirus expressing mCherry fluorescent protein, and grown in bulk. Control CHO cells are defined as ' $m$ Cherry ${ }^{+}$cells'. To generate stable lines expressing mouse CD19, control mCherry ${ }^{+}$cells were infected with a retrovirus expressing CD19 and grown in bulk. Emitter CHO cells expressing CD19 are defined as 'CD19/mCherry ${ }^{+}$cells'.

\section{Induction of SGNTV by co-culture with CD19 cells}

SNTGV/H2Bmcit cells were co-cultured with $\mathrm{CD} 19^{+} / \mathrm{mCherry}^{+}$cells at $1: 1$ ratio in 24-well plates. Cells were incubated with the S3 inhibitor DAPT (10 $\mathrm{\mu M}$; D5942, Sigma-Aldrich) for $30 \mathrm{~h}$ to synchronize the timing of induction, and collected at 12, 24 and $48 \mathrm{~h}$ after removal of DAPT.

\section{Western blot}

Cells were lysed for $5 \mathrm{~min}$ at room temperature in a lysis buffer $[25 \mathrm{mM}$ Tris- $\mathrm{HCl}$ ( $\mathrm{pH} 7.6), 150 \mathrm{mM}$ sodium chloride, $1 \%$ sodium deoxycholate, $1 \%$ Nonidet P-40, $0.1 \%$ sodium dodecyl sulfate (SDS)], supplemented with a protease inhibitor cocktail (Roche). Cellular extracts were boiled for $5 \mathrm{~min}$ and centrifuged at $14,000 \mathrm{rpm}(20,000 \mathrm{~g})$ for $5 \mathrm{~min}$. Equal protein amounts were subjected to SDS-polyacrylamide gel electrophoresis and transferred to nylon membranes. The membranes were incubated for $1 \mathrm{~h} \mathrm{in}$ blocking buffer ( $3 \%$ dry skimmed milk in Tris-buffered saline plus $0.3 \%$ Triton X-100, TBST), incubated for $2 \mathrm{~h}$ with a rabbit anti-GFP antibody (AB3080, Millipore; 1:750 in blocking buffer), washed three times for $10 \mathrm{~min}$ in TBST buffer, incubated for $2 \mathrm{~h}$ with a peroxidase-conjugated goat anti-rabbit IgG antibody (1707515, Bio-Rad; 1:2000 in blocking buffer), and washed three times for $10 \mathrm{~min}$ in TBST buffer. Protein expression was detected by chemiluminescence autoradiography. Blotting membranes were stripped and processed for $\beta$-tubulin as a loading control (mouse anti- $\beta$-tubulin, T8328, Sigma-Aldrich; 1:2000). Incubations and washes were all performed at room temperature.

\section{Flow cytometry analysis}

Co-cultured cells were trypsinized from the plate, diluted in PBS, fixed in $4 \%$ paraformaldehyde, and analyzed for FITC and mCherry fluorescence using a BD LSR II flow cytometer with standard protocols. Relative total fluorescence intensity in Q3 (bottom right quadrant) was quantified by multiplying percentage of cells with FITC MFI in Q3.

\section{$\mathbf{S 2}$ and $\mathbf{S} 3$ inhibitors}

For inhibitor treatment, batimastat (BB94, $50 \mu \mathrm{M}$; SML0041, SigmaAldrich), GM6001 (50 $\mu$ M; SC203979, Santa Cruz Biotechnology), TAPI $(100 \mu \mathrm{M}$; SC20585, Santa Cruz Biotechnology) and DAPT $(10 \mu \mathrm{M})$ were added into growth medium when co-cultured cells were plated. Co-cultured cells were collected $48 \mathrm{~h}$ after the cells were plated.

\section{Induction by substrate-attached ligand on ELISA plates and image analysis}

Rabbit anti-rat IgG $\mathrm{F}\left(\mathrm{ab}^{\prime}\right)_{2}$ (312-005-047, Jackson ImmunoResearch) and anti-goat $\operatorname{IgG}$ (BA-5000, Vector Laboratories) antibodies were diluted at different concentrations in PBS. Diluted antibodies were used to coat 96-well ELISA plates (442404, Thermo Fisher Scientific) at $4{ }^{\circ} \mathrm{C}$ overnight. Next day, 
the ELISA plates were gently washed by PBS, $10 \mathrm{mg} / \mathrm{ml}$ bovine serum albumin was added to block the plates at $37^{\circ} \mathrm{C}$ for $1 \mathrm{~h}$, and SNTGV/UAS$\mathrm{H} 2 \mathrm{Bmcit}$ cells $\left(2 \times 10^{4}\right)$ were then plated. Images were taken under an inverted fluorescence microscope with $10 \times$ objective lenses. RFU of each image was calculated by analyzing the integrated density of all pixels using ImageJ.

Statistical significance was determined using a two-tailed unpaired test ( $t$-test). Data are shown as mean \pm s.e.m. In all cases, three replicates of the experiments were analyzed $(n=3)$.

\section{Transgenic flies}

For all experiments using transgenic flies we modified the SNTGV receptor described above for in vitro experiments and we generated a receptor called SNTG4, which contains the following: (1) SCAD, (2) Drosophila Notch NRR and Notch TMD (residues 1460 to 1767), and the transcriptional regulator GAL4esn (defined as G4 thereafter). GAL4esn includes the DNAbinding domain and the transactivation domain, but it lacks the GAL80binding domain (Sprinzak et al., 2010).

For elav-SNTG4, we introduced the $S N T G 4$ receptor in a pCasper vector containing the 3.5-kb fragment of the elav promoter (Yao and White, 1991). Transgenic elav-SNTG4 flies were produced by standard P-element integration. elav-SNTG4 transgenic flies were screened by GAL4 immunostaining, and the lines with the highest expression level of SNTG4 were chosen for future experiments. The alrm-SNTG4 construct was generated by amplifying a 4973 bp region of alrm promoter from alrmGal4 cassette (Doherty et al., 2009), which then replaced the elav promoter in pCasper-elav-SNTG4. Transgenic alrm-SNTG4 flies were produced by standard P-element integration. alrm-SNTG4 transgenic flies were screened by GAL4 immunostaining, and the lines with the optimal expression level of SNTG4 were chosen for future experiments.

For transgenic flies, we modified the CD19 ligand to carry the CD19 ECD and TMD fused to the red fluorescent marker mCherry to allow identification of the emitter cells. In addition, we included an endocytosis signal from the human LDL receptor (Chen et al., 1990), as endocytosis is thought to be necessary to generate the pulling force that opens the NRR (Meloty-Kapella et al., 2012). The ligand containing CD19, mCherry, and the LDL endocytosis signal are referred to as CD19mch. The sequences of SNTG4 and CD19mch are available in the supplementary Materials and Methods. We compared the activity of SNTGV (with Gal4VP16, human notch NRR and TMD) and SNTG4 (with GAL4esn, Drosophila NRR and TMD) receptors, as well as CD19 and CD19mch ligand, in CHO cells, and we observed that the level of inducibility (maximal induction levels normalized to ligand-independent background levels) were highly similar for these two receptors and two ligands (data not shown).

For LexAop-CD19mch, the CD19mch ligand was cloned in the LexAop pJFRC19 plasmid (Addgene). Transgenic LexAop-CD19mch flies were produced by attb site-specific integration in attP2 site. The drivers repoLexA::GAD and alrm-LexA::GAD, and the reporters 5xUAS-mCD8::GFP and $5 x U A S-C D 4:: t d G F P$ were a gift from Marc Freeman, University of Massachusetts Medical School (MA, USA). Pdf-LexA were a gift from Quan Yuan (National Institute of Neurological Disorders and Stroke, Bethesda, MD, USA). MB247-LexA::VP16 was a gift from Tzumin Lee, Janelia Research Campus, HHMI (VA, USA).

Genotypes of flies analyzed in the figures:

Fig. 3B and Fig. S2a: 5XUAS-CD4::tdGFP/elav-SNTG4; alrm-LexA:: GAD/LexAop-CD19mch

Fig. 3C, Fig. 4 and Fig. S2b: 5XUAS-CD4::tdGFP/elav-SNTG4; repoLexA::GAD/LexAop-CD19mch

Fig. 3D: 5XUAS-CD4::tdGFP/elav-SNTG4; LexAop-CD19mch/TM3

Fig. 5A (top-right, top-left and bottom-left panels), Fig. 5B,D and Fig. 7C (bottom panel): 5XUAS-mCD8::GFP/alrm-SNTG4; MB247-LexA:: VP16/LexAop-CD19mch

Fig. 5A (bottom-right) and Fig. 6B: 5XUAS-mCD8::GFP/alrm-SNTG4; LexAop-CD19mch/TM3

Fig. 5C: 5XUAS-mCD8::GFP/CyO; MB247-LexA::VP16/LexAopCD19mch

Fig. 6C and Fig. 7A (left two panels), Fig. 7B (top panel) and Fig. 7C (top panel): 5XUAS-mCD8::GFP/alrm-SNTG4; Orco-LexA::VP16/LexAopCD19mch
Fig. 6D and Fig. 7A (right two panels): 5XUAS-mCD8::GFP/alrmSNTG4; Pdf-LexA/LexAop-CD19mch

All the crosses were maintained at room temperature, and were repeated at least three times.

\section{Immunostaining and microscopy of fly brain}

The brains of the wandering larvae were dissected in $1 \times$ PBS under a dissection microscope. Brains were fixed by immersing them in a $4 \%$ paraformaldehyde solution in PBS for 15 min at room temperature. Brains were washed in PBS three times for $10 \mathrm{~min}$ each, followed by permeabilization with $\mathrm{PBS} / 0.5 \%$ Triton $\mathrm{X}-100$ (PBST) for $30 \mathrm{~min}$ and blocking with $5 \%$ serum in PBST for $30 \mathrm{~min}$. The brain samples were stained with antibodies against GFP (rabbit polyclonal from Millipore, AB3080; 1:1000), mCherry (rat monoclonal, 5F8, from Chromotek; 1:1000), Repo [mouse monoclonal, 8D12, from Developmental Studies Hybridoma Bank (DSHB); 1:10] and Brp (mouse monoclonal, nc82, from DSHB; 1:50) diluted in $5 \%$ serum/PBST. Brains were incubated with primary antibodies overnight at $4{ }^{\circ} \mathrm{C}$, washed three times in PBST, incubated with goat secondary antibodies (Life Technologies: anti-rabbit Alexa 488, A11008; anti-rat Alexa 555, A21434; anti-mouse Alexa 647, A21236; 1:500) for $2 \mathrm{~h}$ at room temperature (except for Repo and Brp, for which secondary incubation was at $4^{\circ} \mathrm{C}$ overnight), washed in PBST and mounted on glass slides with a clearing solution (Slowfade Gold antifade reagent, Invitrogen).

Stained brains were imaged with confocal microscopes (Olympus Fluoview 300 or Zeiss 710 ) under a $40 \times$ or $60 \times$ objective. In a typical experiment, we imaged 150 sections with an optical thickness of 0.3-0.5 $\mu \mathrm{m}$ from dorsal or ventral sides. Confocal stacks were processed with Fiji to obtain maximal projections.

\section{Acknowledgements}

Michael Elowitz provided the CHO-UAS-H2Bmcit and CHO-rat Delta cell lines James Kochenderfer (National Cancer Institute, Bethesda, MD, USA) supplied the 1D3-28z.1-3 and the MSGV-CD19 plasmids. Marc Freeman provided the repoLexA::GAD and alrm-LexA::GAD drivers. Wilm Stork, Lukas Neukomm, Marc Freeman and Elizabeth Hong provided advice on the Drosophila nervous system anatomy and genetics. Daniel Lee helped edit the manuscript. Imaging from Zeiss 710 was performed in the Biological Imaging Facility, with the support of the Caltech Beckman Institute and the Arnold and Mabel Beckman Foundation.

\section{Competing interests}

The authors declare no competing or financial interests.

\section{Author contributions}

C.L. conceived the project. C.L. and T.-H.H. designed experiments. T.-H.H. performed all experiments with cell culture and transgenic Drosophila. T.V. performed western blot analysis. T.-H.H. and C.L. wrote the manuscript.

\section{Funding}

This work was funded by the National Institutes of Health [1R21NS08485 to C.L.]. Deposited in PMC for release after 12 months.

\section{Supplementary information}

Supplementary information available online at

http://dev.biologists.org/lookup/doi/10.1242/dev.142406.supplemental

\section{References}

Anderson, K. V. and Ingham, P. W. (2003). The transformation of the model organism: a decade of developmental genetics. Nat. Genet. 33 Suppl., 285-293.

Artavanis-Tsakonas, S. and Muskavitch, M. A. T. (2010). Notch: the past, the present, and the future. Curr. Top. Dev. Biol. 92, 1-29.

Barnea, G., Strapps, W., Herrada, G., Berman, Y., Ong, J., Kloss, B., Axel, R. and Lee, K. J. (2008). The genetic design of signaling cascades to record receptor activation. Proc. Natl. Acad. Sci. USA 105, 64-69.

Bellen, H. J., Tong, C. and Tsuda, H. (2010). 100 years of Drosophila research and its impact on vertebrate neuroscience: a history lesson for the future. Nat. Rev. Neurosci. 11, 514-522.

Bowtell, D. D., Lila, T., Michael, W. M., Hackett, D. and Rubin, G. M. (1991). Analysis of the enhancer element that controls expression of sevenless in the developing Drosophila eye. Proc. Natl. Acad. Sci. USA 88, 6853-6857.

Brou, C., Logeat, F., Gupta, N., Bessia, C., LeBail, O., Doedens, J. R., Cumano A., Roux, P., Black, R. A. and Israel, A. (2000). A novel proteolytic cleavage 
involved in Notch signaling: the role of the disintegrin-metalloprotease TACE. Mol. Cell 5, 207-216.

Chen, W. J., Goldstein, J. L. and Brown, M. S. (1990). NPXY, a sequence often found in cytoplasmic tails, is required for coated pit-mediated internalization of the low density lipoprotein receptor. J. Biol. Chem. 265, 3116-3123.

del Valle Rodriguez, A., Didiano, D. and Desplan, C. (2011). Power tools for gene expression and clonal analysis in Drosophila. Nat. Methods 9, 47-55

Denk, W. and Horstmann, H. (2004). Serial block-face scanning electron microscopy to reconstruct three-dimensional tissue nanostructure. PLoS Biol. 2 , e329.

Doherty, J., Logan, M. A., Taşdemir, O. E. and Freeman, M. R. (2009) Ensheathing glia function as phagocytes in the adult Drosophila brain. J. Neurosci. 29, 4768-4781.

Edwards, T. N. and Meinertzhagen, I. A. (2010). The functional organisation of glia in the adult brain of Drosophila and other insects. Prog. Neurobiol. 90, 471-497.

Enquist, L. W. and Card, J. P. (2003). Recent advances in the use of neurotropic viruses for circuit analysis. Curr. Opin. Neurobiol. 13, 603-606.

Feinberg, E. H., VanHoven, M. K., Bendesky, A., Wang, G., Fetter, R. D., Shen, K. and Bargmann, C. I. (2008). GFP Reconstitution Across Synaptic Partners (GRASP) defines cell contacts and synapses in living nervous systems. Neuron 57, 353-363.

Fetcho, J. R. and Liu, K. S. (1998). Zebrafish as a model system for studying neuronal circuits and behavior. Ann. N. Y. Acad. Sci. 860, 333-345.

Freeman, M. R. (2015). Drosophila central nervous system glia. Cold Spring Harb. Perspect. Biol. 7, pii: a020552.

Freeman, M. R., Delrow, J., Kim, J., Johnson, E. and Doe, C. Q. (2003) Unwrapping glial biology: $\mathrm{Gcm}$ target genes regulating glial development diversification, and function. Neuron 38, 567-580

Gordon, W. R., Vardar-Ulu, D., Histen, G., Sanchez-Irizarry, C., Aster, J. C. and Blacklow, S. C. (2007). Structural basis for autoinhibition of Notch. Nat. Struct Mol. Biol. 14, 295-300.

Gordon, W. R., Zimmerman, B., He, L., Miles, L. J., Huang, J., Tiyanont, K., McArthur, D. G., Aster, J. C., Perrimon, N., Loparo, J. J. et al. (2015) Mechanical allostery: evidence for a force requirement in the proteolytic activation of Notch. Dev. Cell 33, 729-736.

Griesbeck, O., Baird, G. S., Campbell, R. E., Zacharias, D. A. and Tsien, R. Y (2001). Reducing the environmental sensitivity of yellow fluorescent protein Mechanism and applications. J. Biol. Chem. 276, 29188-29194.

Helfrich-Forster, C. (1997). Development of pigment-dispersing hormoneimmunoreactive neurons in the nervous system of Drosophila melanogaster. J. Comp. Neurol. 380, 335-354.

Jagadish, S., Barnea, G., Clandinin, T. R. and Axel, R. (2014). Identifying functional connections of the inner photoreceptors in Drosophila using TangoTrace. Neuron 83, 630-644.

Kochenderfer, J. N., Feldman, S. A., Zhao, Y., Xu, H., Black, M. A., Morgan, R. A., Wilson, W. H. and Rosenberg, S. A. (2009). Construction and preclinical evaluation of an anti-CD19 chimeric antigen receptor. J. Immunother. 32, 689-702.

Kopan, R. and Ilagan, M. X. G. (2009). The canonical Notch signaling pathway: unfolding the activation mechanism. Cell 137, 216-233.

Lewandoski, M. (2001). Conditional control of gene expression in the mouse. Nat Rev. Genet. 2, 743-755.

Lichtman, J. W. and Denk, W. (2011). The big and the small: challenges of imaging the brain's circuits. Science $334,618-623$.

Lin, C.-W., Sim, S., Ainsworth, A., Okada, M., Kelsch, W. and Lois, C. (2010). Genetically increased cell-intrinsic excitability enhances neuronal integration into adult brain circuits. Neuron $65,32-39$

Lois, C., Hong, E. J., Pease, S., Brown, E. J. and Baltimore, D. (2002). Germline transmission and tissue-specific expression of transgenes delivered by lentiviral vectors. Science 295, 868-872.

Meinertzhagen, I. A. and Lee, C.-H. (2012). The genetic analysis of functional connectomics in Drosophila. Adv. Genet. 80, 99-151.

Meloty-Kapella, L., Shergill, B., Kuon, J., Botvinick, E. and Weinmaster, G. (2012). Notch ligand endocytosis generates mechanical pulling force dependent on dynamin, epsins, and actin. Dev. Cell 22, 1299-1312.

Morsut, L., Roybal, K. T., Xiong, X., Gordley, R. M., Coyle, S. M., Thomson, M. and Lim, W. A. (2016). Engineering customized cell sensing and response behaviors using synthetic Notch receptors. Cell 164, 780-791.

Mumm, J. S., Schroeter, E. H., Saxena, M. T., Griesemer, A., Tian, X., Pan, D. J., Ray, W. J. and Kopan, R. (2000). A ligand-induced extracellular cleavage regulates gamma-secretase-like proteolytic activation of Notch1. Mol. Cell $\mathbf{5}$, 197-206.
Nagai, Y., Sano, H. and Yokoi, M. (2005). Transgenic expression of Cre recombinase in mitral/tufted cells of the olfactory bulb. Genesis 43, 12-16.

Nichols, J. T., Miyamoto, A., Olsen, S. L., D'Souza, B., Yao, C. and Weinmaster, G. (2007). DSL ligand endocytosis physically dissociates Notch1 heterodimers before activating proteolysis can occur. J. Cell Biol. 176, 445-458.

Oberdick, J., Smeyne, R. J., Mann, J. R., Zackson, S. and Morgan, J. I. (1990). A promoter that drives transgene expression in cerebellar Purkinje and retinal bipolar neurons. Science 248, 223-226.

Omoto, J. J., Yogi, P. and Hartenstein, V. (2015). Origin and development of neuropil glia of the Drosophila larval and adult brain: two distinct glial populations derived from separate progenitors. Dev. Biol. 404, 2-20.

Peca, J. and Feng, G. (2012). Cellular and synaptic network defects in autism. Curr. Opin. Neurobiol. 22, 866-872.

Roybal, K. T., Rupp, L. J., Morsut, L., Walker, W. J., McNally, K. A., Park, J. S and Lim, W. A. (2016). Precision tumor recognition by T cells with combinatorial antigen-sensing circuits. Cell 164, 770-779.

Sheng, M. (2001). Molecular organization of the postsynaptic specialization. Proc Natl. Acad. Sci. USA 98, 7058-7061.

Sprecher, S. G., Cardona, A. and Hartenstein, V. (2011). The Drosophila larval visual system: high-resolution analysis of a simple visual neuropil. Dev. Biol. 358 33-43

Sprinzak, D., Lakhanpal, A., LeBon, L., Santat, L. A., Fontes, M. E., Anderson, G. A., Garcia-Ojalvo, J. and Elowitz, M. B. (2010). Cis-interactions between Notch and Delta generate mutually exclusive signalling states. Nature 465, 86-90. Stephenson, N. L. and Avis, J. M. (2012). Direct observation of proteolytic cleavage at the $\mathrm{S} 2$ site upon forced unfolding of the Notch negative regulatory region. Proc. Natl. Acad. Sci. USA 109, E2757-E2765.

Stocker, R. F., Heimbeck, G., Gendre, N. and de Belle, J. S. (1997). Neuroblas ablation in Drosophila P[GAL4] lines reveals origins of olfactory interneurons. J. Neurobiol. 32, 443-456.

Stork, T., Bernardos, R. and Freeman, M. R. (2012). Analysis of glial cell development and function in Drosophila. Cold Spring Harb. Protoc. 2012, 1-17.

Struhl, G. and Adachi, A. (1998). Nuclear access and action of notch in vivo. Cell 93, 649-660.

Struhl, G. and Adachi, A. (2000). Requirements for presenilin-dependent cleavage of notch and other transmembrane proteins. Mol. Cell 6, 625-636.

Sudhof, T. C. (2012). The presynaptic active zone. Neuron 75, 11-25

Talsma, A. D., Christov, C. P., Terriente-Felix, A., Linneweber, G. A., Perea, D. Wayland, M., Shafer, O. T. and Miguel-Aliaga, I. (2012). Remote control of renal physiology by the intestinal neuropeptide pigment-dispersing factor in Drosophila. Proc. Natl. Acad. Sci. USA 109, 12177-12182.

Tian, L., Akerboom, J., Schreiter, E. R. and Looger, L. L. (2012). Neural activity imaging with genetically encoded calcium indicators. Prog. Brain Res. 196, 79-94.

Tiyanont, K., Wales, T. E., Aste-Amezaga, M., Aster, J. C., Engen, J. R. and Blacklow, S. C. (2011). Evidence for increased exposure of the Notch1 metalloprotease cleavage site upon conversion to an activated conformation. Structure 19, 546-554

Varnum-Finney, B., Wu, L., Yu, M., Brashem-Stein, C., Staats, S., Flowers, D. Griffin, J. D. and Bernstein, I. D. (2000). Immobilization of Notch ligand, Delta-1, is required for induction of notch signaling. J. Cell Sci. 113, 4313-4318.

Venken, K. J. T., Simpson, J. H. and Bellen, H. J. (2011). Genetic manipulation of genes and cells in the nervous system of the fruit fly. Neuron 72, 202-230.

Vooijs, M., Ong, C.-T., Hadland, B., Huppert, S., Liu, Z., Korving, J., van den Born, M., Stappenbeck, T., Wu, Y., Clevers, H. et al. (2007). Mapping the consequence of Notch1 proteolysis in vivo with NIP-CRE. Development 134 535-544.

Wang, W., Goswami, S., Sahai, E., Wyckoff, J. B., Segall, J. E. and Condeelis, J. S. (2005). Tumor cells caught in the act of invading: their strategy for enhanced cell motility. Trends Cell Biol. 15, 138-145.

Wickersham, I. R., Lyon, D. C., Barnard, R. J. O., Mori, T., Finke, S., Conzelmann, K.-K., Young, J. A. T. and Callaway, E. M. (2007) Monosynaptic restriction of transsynaptic tracing from single, genetically targeted neurons. Neuron 53, 639-647.

Yao, K. M. and White, K. (1991). Organizational analysis of elav gene and functional analysis of ELAV protein of Drosophila melanogaster and Drosophila virilis. Mol. Cell. Biol. 11, 2994-3000.

Yizhar, O., Fenno, L. E., Davidson, T. J., Mogri, M. and Deisseroth, K. (2011) Optogenetics in neural systems. Neuron 71, 9-34

Zhang, W., Yan, Z., Li, B., Jan, L. Y. and Jan, Y. N. (2014). Identification of moto neurons and a mechanosensitive sensory neuron in the defecation circuitry of Drosophila larvae. Elife 3, e03293.

Zwarts, L., Van Eijs, F. and Callaerts, P. (2015). Glia in Drosophila behavior. J. Comp. Physiol. A Neuroethol. Sens. Neural Behav. Physiol. 201, 879-893. 